\title{
A Novel Network Intrusion Attempts Prediction Model Based on Fuzzy Neural Network*
}

\author{
Guiling Zhang and Jizhou Sun \\ Department of Electronic Information Engineering, Tianjin University, 300072, China \\ glzhang808@sohu.com, jzsunatju.edu.cn
}

\begin{abstract}
Identifying the intrusion attempts of the monitored systems is extremely vital for the next generation intrusion detection system. In this paper, a novel network intrusion attempts prediction model (FNNIP) is developed, which is based on the observation of network packet sequences. A new fuzzy neural network based on a novel BP learning algorithm is designed and then applied to the network intrusion attempts predicting scheme. After given the analysis of the features of the experimental data sets, the experiment process is detailed. The experimental results show that the proposed Scheme has good accuracy of predicting the network intrusion attempts by observing the network packet sequences.
\end{abstract}

Keywords: Intrusion Attempt Prediction, Intrusion Detection, Fuzzy Neural Network.

\section{Introduction}

Intrusion detection techniques have become an important research area in modern information security system. Denning first proposes a model for building a real time intrusion detection expert system by analyzing the profiles representing the system behavior from audit records [1]. S. Forrest and others propose an intrusion detection model based on short sequences of system calls [2]. W. Lee and others build a datamining framework for intrusion detection system $[4,5]$. H. Jin and coworkers extends the W. Lee's work by applying fuzzy data mining algorithm to intrusion detection [3]. Paper [6] and paper [7] apply neural networks to building intrusion detection models. The above methods are only able to detect intrusions after the attacks have occurred, either partially or fully, which makes it difficult to contain or stop the attack in real time [9]. So it is necessary to tap intrusion attempts prediction techniques into IDS.

$\mathrm{N}$. Ye and others proposed two methods of forecasting normal activities in computer systems for intrusion detection. Their method provides performance improvement on intrusion detection [8]. L. Feng and coworkers apply a plan recognition method for predicting the anomaly events and the intensions of possible intruders to a computer system based on the observation of system call sequences [9].

This paper extends their works by observing network packet sequences to predict intrusion attempts. We develop a new intrusion attempts prediction model based on

This research was supported by the National High Technology Development 863 program of China under Grant No. 2002AA142010. 
fuzzy neural network (FNNIP) for network intrusion detection system. Only the critical features in the network connection records are employed to describe the actions of the normal or attacks. The experimental results show that the proposed Scheme has good accuracy of predicting the network intrusion attempts by observing the network packet sequences.

The rest of this paper is organized as follows: How to construct the structure of the proposed FNNIP is discussed in section 2. In this section, a new BP learning algorithm is also designed for the proposed scheme. Section 3 details the description of the network traffic behaviors. Some experiments are described in section 4. In section 5, some conclusions and future works are given.

\section{The Structure of Fuzzy Neural Network for Network Intrusion Attempts Prediction}

The structure of the proposed fuzzy neural network based network intrusion attempts prediction (FNNIP) system is designed in Fig.1 [12].

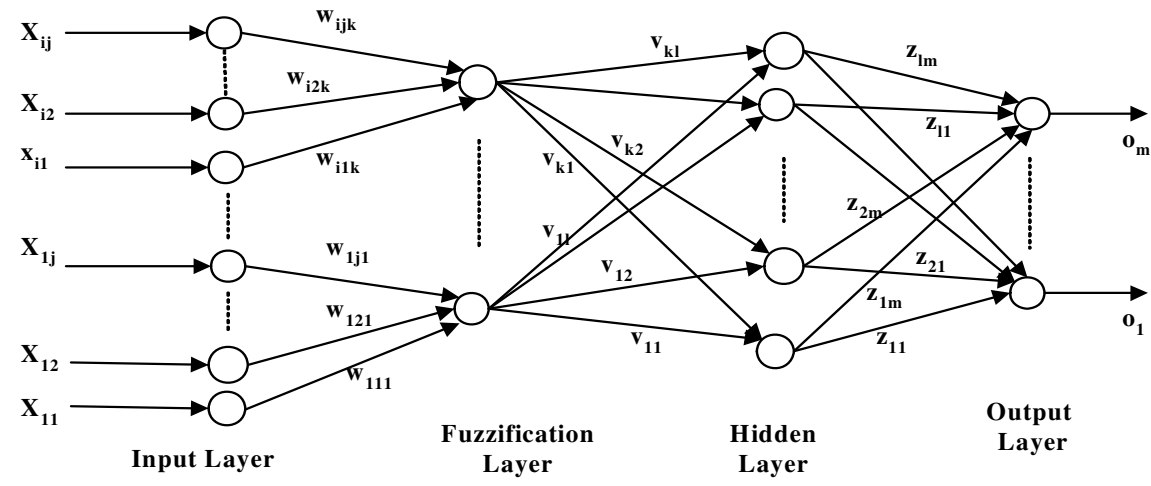

Fig. 1. The Structure of The Proposed FNNIP

The input layer consists of $\mathrm{N}$ input vectors $\mathbf{x}_{i}, i=1,2, \cdots, N-1, N$; each vector $\mathbf{x}_{i}$ has $t_{j}$ elements. The total of the input nodes are $N \times t_{j}$.

The fuzzification layer has $\mathrm{t}_{\mathrm{f}}=\mathrm{N}$ nodes. Each node in the fuzzification layer represents a fuzzy membership function for the $t_{j}$ input variable in vector $\mathbf{x}_{i}$. The fuzzy membership function used is described in equation (1).

$$
\mu_{i j_{k}}\left(x_{i j}\right)=e^{-\left(\sum_{j=1}^{t_{j}} w_{i j k} x_{i j}\right)^{2}}
$$


Where $\mu_{i j k}$ is the $k^{\text {th }}$ output of the fuzzification layer, $w_{i j k}$ is the weight between input nodes $x_{i j}$ and the $k^{\text {th }}$ fuzzification nodes.

For each node in the hidden layer, the inputs are $\mu_{i j k}$ and the outputs $h_{l}$ are as follows [13]:

$$
h_{l}=f\left(\text { net }_{l}\right) \quad n e t_{l}=\sum_{k=1}^{t_{f}} v_{k l} \mu_{i j_{k}}\left(x_{i j}\right)
$$

Where $v_{k l}$ are the weights between the fuzzification layer and the hidden layer.

In the output layer, each output $o_{m}$ is as follows:

$$
o_{m}=f\left(\text { net }_{m}\right) \quad n e t_{m}=\sum_{l=0}^{t_{h}} z_{l m} h_{l}
$$

Where $t_{h}$ is the number of nodes in the hidden layer, $z_{l m}$ are the weights between the hidden layer and the output layer and $f($ net $)=1 / 1+e^{-x}$.

The error function of the FNNIP is:

$$
E=\frac{1}{2} \sum_{m}\left(t_{m}-o_{m}\right)^{2}
$$

Where $t_{m}$ is the expected output for the $m^{t h}$ output node $o_{m}$. The weights between hidden layer and output layer are adjusted by BP algorithm as follows [12]:

$$
\begin{gathered}
z_{l m}(n)=z_{l m}(n-1)+\Delta z_{l m}(n) \\
\text { Where } \quad \Delta z_{i l m}(n)=\eta \delta_{m} h_{l}+\alpha \Delta z_{l m}(n-1)
\end{gathered}
$$

Where $\mathrm{n}$ is the iteration count, $\delta_{m}=t_{m}-o_{m}$. The weights between fuzzification layer and hidden layer are adjusted by the following equations [12]:

$$
\begin{gathered}
v_{k l}(n)=v_{k l}(n-1)+\Delta v_{k l}(n) \\
\text { Where } \quad \Delta v_{k l}(n)=\eta \delta_{k l} \mu_{i j k}+\alpha \Delta v_{k l}(n-1) \\
\delta_{k l}=\left(\prod_{k=1}^{t_{f}} \mu_{i j k}\right)\left(\sum_{m=1}^{t_{o}} \delta_{m} z_{l m} / \mu_{i j k}\right)
\end{gathered}
$$

Where $t_{o}$ is the total number of the output nodes. Similarly, the weights between input layer and fuzzification layer are adjusted as follows [12, 13]:

$$
w_{i j k}(n)=w_{i j k}(n-1)+\Delta w_{i j k}(n)
$$




$$
\begin{gathered}
\text { Where } \quad \Delta w_{i j k}(n)=\eta \delta_{k l} \mu_{0}+\alpha \Delta w_{i j k}(n-1) \\
\mu_{0}=\frac{1}{t_{j}} \sum_{j=1}^{t_{j}} \exp \left(-\left(w_{i j k} x_{i j k}\right)^{2}\right.
\end{gathered}
$$

In the formula above, " $\eta$ " is a learning rate, which controls the rate of convergence, in the beginning of training it has the larger value, then decrease quickly [13]. We set $\eta=b_{2}\left(1-t / t_{m}\right), b_{2}$ is a constant value and in the range of [0,1] (it is set to 0.3 in this paper), $t_{m}$ is the maximal training number in advance, $\mathrm{t}$ is the $t^{\text {th }}$ training. The momentum constant " $\alpha$ " (kept at 0.6 throughout) is able to add to speed up the training process and avoid local minima. The initial weights are randomly selected in the interval $[-1,+1]$. The training process is continued till $E(n)<\mathcal{E}$ at all points or the number of iteration reaches its maximum (e.g., 5000). The value of $\varepsilon$ is taken to be $1 \times e^{-4}$ during training.

\section{The Description of the Network Traffic Behaviors}

In this paper, we assume that the current state of network connection record dependents on the latest $t_{j}$ continuous network connection records sequences. So, the network intrusion prediction method is defined as:

Definition 1. Given the latest $t_{j}$ continuous network connection records sequences $\mathbf{X}$, each record $\mathbf{x}_{\mathbf{i}}$ in $\mathbf{X}$ has $\mathrm{N}-1$ connection features and 1 label feature (normal or abnormal). The next network connection record $\mathbf{x}_{\text {next }}$ is predicted by the given latest $\mathbf{t}_{\mathbf{j}}$ serial network connection records, and the label feature (normal or abnormal) may also be predicted:

$$
\mathbf{X}=\left(\mathbf{x}_{1}, \mathbf{x}_{2}, \cdots, \mathbf{x}_{t_{j}}\right) \Rightarrow \mathbf{x}_{\text {next }}
$$

According to definition 1, it is assumed that the next network action can be correctly predicted by the latest continuous network actions.

To improve the performance of the FNNIP, we should reduce the dimensions of the input vector. Some important features for intrusion prediction system are employed. Before we analyze the data set, data standardization must be performed. The data standardization process is described as follows [3]:

$$
x_{i k}^{\prime}=\frac{x_{i k}-\overline{x_{k}}}{s_{k}},(i=1,2, \ldots, n, k=1,2, \ldots, m)
$$

Where $\overline{x_{k}}$ and $s_{k}$ is the mean value and standard deviation of one feature or the kth dimension of data set [3]. 


$$
\begin{gathered}
\bar{x}_{k}=\frac{1}{n} \sum_{i=1}^{n} x_{i k} \\
s_{k}=\sqrt{\frac{1}{n} \sum_{i=1}^{n}\left(x_{i k}-\overline{x_{k}}\right)^{2}}
\end{gathered}
$$

This procedure transforms the mean of the set of feature values to zero and the standard deviation to one. But the $x_{i k}^{\prime}$ may not be in the interval $[0,1]$ and it is processed as follows [3]:

$$
x_{i k}^{\prime \prime}=\frac{x_{i k}^{\prime}-\min _{1 \leq i \leq n}\left\{x_{i k}^{\prime}\right\}}{\max _{1 \leq i \leq n}\left\{x_{i k}^{\prime}\right\}-\min _{1 \leq i \leq n}\left\{x_{i k}^{\prime}\right\}},(k=1,2, \ldots, m)
$$

To select the important features, we calculate the correlation coefficient between $x_{i}$ and $x_{j}$ as follows [3]:

$$
r_{i j}=\frac{\sum_{k=1}^{m}\left|x_{i k}-\overline{x_{i}} \| x_{j k}-\overline{x_{j}}\right|}{\sqrt{\sum_{k=1}^{m}\left(x_{i k}-\overline{x_{i}}\right)^{2}} \cdot \sqrt{\sum_{k=1}^{m}\left(x_{j k}-\overline{x_{j}}\right)^{2}}}
$$

It is strong negative correlation between $x_{i}$ and $x_{j}$ when $r_{i j}=-1$ and it is strong positive correlation between $x_{i}$ and $x_{j}$ when $r_{i j}=1$.

In this paper, we compute the correlation coefficients between two features by the soft ware SPSS [3]. If the $r_{i j} \geq 0.7$, we select only one of them to represent these two fields. The selected features are describe as follows:

$$
\begin{aligned}
& \mathbf{x}=\left(x_{1}, x_{2}, \cdots, x_{N}\right)=\left(\text { duration, service, flag, src _ bytes }, d_{s} t_{-}\right. \text {bytes, wrong _ } \\
& \text { fragment, urgent, hot, num _ failed_logins, su_attempted, num _ root, num _ } \\
& \text { file_creations, num_access_files, count, srv_count, serror_rate, srv _ serror } \\
& \text { _rate, rerror_rate, srv_rerror_rate, same _ srv _ rate, srv_diff _ host_rate, } \\
& d s t_{-} h o s t_{-} \text {count, dst_host_srv_count, dst_host_same_src _ port_rate, } \\
& d s t_{-} \text {host_srv_diff_host_rate, label) }
\end{aligned}
$$

\section{Experiments}

\subsection{Training the FNNIP}

The proposed FNNIP are evaluated by the famous KDD'99 data sets [10]. There are four main categories attacks in the KDD'99 data sets: DoS, R2L, U2R and Probing.

The training data is randomly collected from the original raw KDD'99 data sets, which contains all of four categories attacks (20\%) and normal records (80\%). 
We select only 17 features from the total 41 features of each connection record and an additional label feature as the inputs of the FNNIP $(\mathrm{N}=18)$. The latest $5\left(t_{j}=5\right.$ for the FNNIP) continuous connection records are employed to predict the next connection records. So, the total input nodes of the FNNIP are 90. A slide window (window size is 5 records and the step is 1) employed covers the training data and its outputs are passed to the FNNIP. The total nodes of fuzzification and the total nodes of output layer are 18, respectively. The total nodes of hidden layer are selected as $\mathrm{H}=30,40,50$, respectively.

During training process, the training data set is exposed to the proposed FNNIP and the weights of the FNNIP are initialized randomly. To adjust the weights, the actual outputs are compared with desired targets, respectively. If the output and target match (in the same range), no change is made to the weights. However, if the output differs from the target (in the different range) a change must be made to the specific weight. The training process is continued till $E(n)<\varepsilon$ at all points or the number of iteration reaches its maximum (e.g., 10000).

\subsection{Testing the FNNIP}

Four groups test data sets are generated from the KDD'99 test data set. It contains a total of 24 attacks in training data and additional 14 types attacks in the test data only. The extracted test data sets contain $80 \%$ normal connection records and $20 \%$ attacks records but not including the training data set. The testing data is also normalized as described in section 3.2. We use the Euclidean distance to measure the similarity between the active output $\mathbf{o}_{o}=\left\{o_{o 1}, o_{o 2}, \cdots, o_{o n-1}\right\}$ and the corresponding records $\mathbf{o}_{t}=\left\{o_{t 1}, o_{t 2}, \cdots, o_{t n-1}\right\}$ in the test data (except the label feature):

$$
d=\sqrt{\sum_{i=1}^{n-1}\left(o_{o i}-o_{t i}\right)^{2}}
$$

If $\mathrm{d}$ is little than a specific value (e.g. 0.3) then the result of prediction of connection record is right, otherwise, the result of prediction is wrong.

For the label feature, if its value of the prediction is larger than 0.5 , the prediction record is abnormal; otherwise, the prediction record is normal.

The hit occurs only when the connection record is correctly predicted and its corresponding label is rightly predicted.

The FNNIP presented in this paper is evaluated using ROC charts. The experimental results are showed in Fig.2 to Fig.5, which are the average results for the four groups testing data set and when the Euclidean distance satisfies $\mathrm{d}<0.3$.

Given a specific number of hidden nodes for the FNNIP, we can obtain a pair of hit rate and false alarm for specific type of attacks. Fig.2 to Fig.5 show that the performance of the FNNIP increases with different numbers of hidden nodes, but the hit rate can only improve slightly after the number of hidden node is greater than 40 . Fig. 2 is the ROC of prediction of the DoS attacks. The hit-rate is greater than $80 \%$ when false alarm rate is $10 \%$ and the number of hidden nodes of FNNIP is greater than 40 . If we allow the false alarm greater than $17 \%$, the hit-rate is greater than $90 \%$.

Fig. 3 is the ROC of prediction of the Probing attacks. The hit-rate is greater than $80 \%$ when false alarm rate is $5 \%$ and the number of hidden nodes of FNNIP is 
greater than 40 . If we agree that the false alarm greater than $15 \%$, the hit-rate is greater than $90 \%$.

Fig. 4 is the ROC of prediction of the U2R attacks. The hit-rate is greater than $80 \%$ when false alarm rate is $5 \%$ and the number of hidden nodes of the FNNIP is greater than $50 \%$. If we permit the false alarm greater than $20 \%$, the hit-rate is greater than $90 \%$.

Fig. 5 is the ROC of predicting the R2L attacks. From Fig.5, The hit-rate is between $70 \%$ and $75 \%$ when false alarm rate is $10 \%$ and the number of hidden nodes of the FNNIP is greater than 40. If we allow the false alarm greater than $30 \%$, the hit-rate may be greater than $90 \%$.

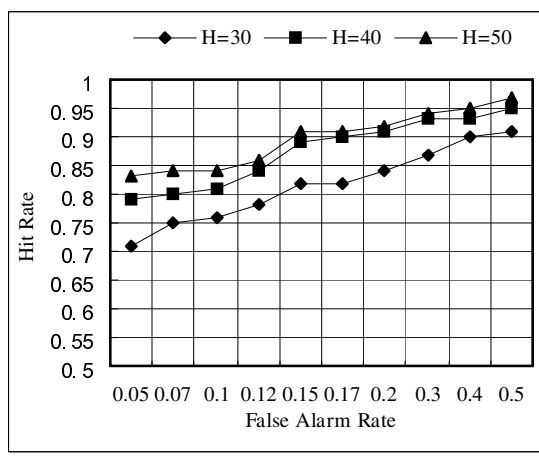

Fig. 2. The ROC for the DoS attacks

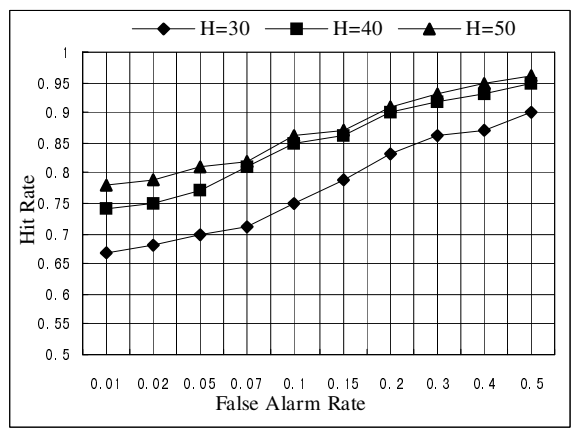

Fig. 4. The ROC for the U2R attacks

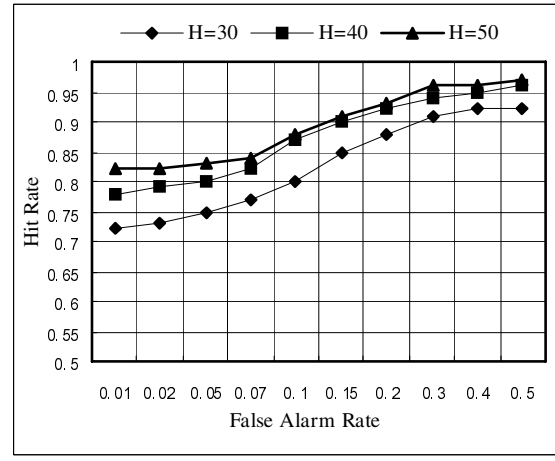

Fig. 3. The ROC for the Probing attacks

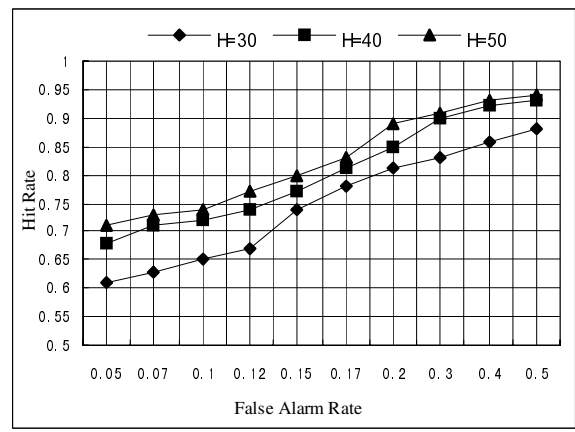

Fig. 5. The ROC for the R2L attacks

From Fig.2 to Fig.5, we can conclude that the DoS and R2L attacks are more flexible than the other two type attacks during their intrusion procedure. It is more difficult to predict the Probing and U2R attacks correctly.

\section{Conclusions and Future Work}

A novel method of predicting intrusion intentions based on fuzzy neural network has been investigated in this paper. The proposed FNNIP use the latest continuous 
connection records and their labels ("normal" or "intrusion") to predict the next record and the corresponding label ("normal" or "intrusion"). It is evaluated by the famous KDD'99 dataset and the experiment results demonstrate that the proposed FNNIP has acceptable ability of predicting different type of attacks in network connection records.

However there are some issues to be studied in the future. First, how much is the optimal number of the latest continuous records to predict the next records? Second, what features are more important to the FNNIP prediction scheme? Final, how much is optimal number of hidden nodes for FNNIP corresponding to the different parameters?

\section{References}

1. Warrender, C., Forrest, S., and Pearlmutter, B.: Detecting Intrusions Using System Calls: Alternative Data Models. Proceedings of the 1999 IEEE Symposium on Security and Privacy May 1999 Page(s) 133 - 145.

2. Forrest, S., Hofmeyr, S.A.; Somayaji, A., Longstaff, T.A.: A Sense of Self for UNIX Processes. Proceedings of the 1996 IEEE Symposium on Security and Privacy May 1996 Page(s) 120 - 128.

3. Hai J., Jianhua S., Hao C., Zongfen H.: A Fuzzy Data Mining Based Intrusion Detection Model. Distributed Computing Systems, 2004. FTDCS 2004. Proceedings. 10th IEEE International Workshop on Future Trends of 26-28 May 2004 Page(s) 191 - 197.

4. Lee, W., Stolfo, S.J.: Data Mining Approaches for Intrusion Detection. Proceedings of the Seventh USENIX Security Symposium, January 1998 Page(s) 79-93.

5. Wenke L., Stolfo, S.J.; Mok, K.W.: A Data Mining Framework for Building Intrusion Detection Models. Proceedings of the 1999 IEEE Symposium on Security and Privacy May 1999 Page(s) 120 - 132.

6. Ghosh, A.K., Schwartzbard, A.: A Study in Using Neural Networks for Anomaly and Misuse Detection. Proceedings of the Eighth USENIX Security Symposium (Security'99), Aug. 1999 Page(s) 141-151.

7. Amini M., Jalili R.: Network-Based Intrusion Detection Using Unsupervised Adaptive Resonance Theory (ART). Advances in Neural Information Processing Systems 10, Cambridge, MA: MIT Press, 1998.

8. Nong, Y., Qiang, C., Borror, C.M.: EWMA Forecast of Normal System Activity for Computer Intrusion Detection. IEEE Transactions on Reliability Volume 53, Issue 4, Dec. 2004 Page(s) 557 - 566.

9. Feng, L., Xiaohong G., Sangang G., Yan G., Peini L.: Predicting the Intrusion Intentions by Observing System Call Sequences. Computers and Security, Elsevier Science, Volume 23, Issue 3, May, 2004 Page(s) 241-252.

10. KDD Cup 1999 data. http://kdd.ics.uci.edu/databases/kddcup99/kddcup99.htm.

11. Mukkamala S., Sung A. H.: Feature Selection for Intrusion Detection Using Neural Networks and Support Vector Machines. Journal of the Transportation Research Board, Transportation Research Record No 1822, 2003 Page(s) 33-39.

12. Dash, P.K., Pradhan, A.K., Panda, G.: A Novel Fuzzy Neural Network Based Distance Relaying Scheme. IEEE Transactions On Power Delivery, VOL. 15, NO. 3, July 2000 Page(s) 902 - 907.

13. Yuan, F., Wu, H., and Yu, G.: Web Users' Classification Using Fuzzy Neural Network. Knowledge-Based Intelligent Information and Engineering Systems: 8th International Conference, Kes 2004, Wellington, New Zealand, September, 2004. 